\title{
DESAGREGACIÓN DE LA ESTRUCTURA DE LA FECUNDIDAD POR EDADES EMPLEANDO LA FUNCIÓN DE GOMPERTZ
}

\author{
Alejandro Mina V. \\ El Colegio de México
}

\section{Introducción}

Сомо сом ponente demográfico, la fecundidad se expresa fundamentalmente vía las "tasas específicas de fecundidad por edades" y de la tasa global de fecundidad (es la suma de las específicas). Esta última (TGF) se entiende como el número medio de hijos por mujer al final del período reproductivo de una cohorte hipotética de mujeres que ha estado sujeta a la fecundidad por edades de un momento determinado.

Las fuentes para obtener la información sobre fecundidad, son los registros de las estad ísticas vitales, las encuestas y los censos, pero debido a la notoria insuficiencia, tanto en cantidad como en calidad de dicha información, no pueden aplicarse confiadamente los métodos tradicionales de estimación. Por ello es conveniente ajustar las irregularidades propias de la información.

Un procedimiento que puede ser de utilidad para realizar ajustes del patrón de fecundidad por edades es presentado en este trabajo; dicho procedimiento fue desarrollado por Juan Chackiel en $1979,{ }^{1}$ su procedimiento está basado en ideas de W. Brass ${ }^{2}$ y V. Kandiah ${ }^{3}$, quienes se apoyaron en la función de Gompertz linealizada mediante una transformación logarítmica.

El objetivo del presente trabajo es presentar el modelo del doble logaritmo de la función de Gompertz, aplicándolo a información ob-

1 Ver: Chackiel, Juan, "Estructura de la fecundidad por edades: ajuste y proyección mediante la función de Gompertz linealizada". Notas de Población. No. 20, agosto 1979.

2 Brass, W.: The relational Gompertz model of fertility by age of woman. (Inédito). Brass, W.: An application of the relational Gompertz model of fertility. (Inédito).

3 Kandiah, V.: The use of the relational fertility model parameters in population projections. East-West Center, Honolulu. (Inédito). 
tenida de la Encuesta Mexicana de Fecundidad levantada, a nivel nacional, en el año de 1976, y señalar el empleo de dicho modelo en la desagregación de grupos de edades quinquenales a edades individuales, de la estructura por edad de la fecundidad.

2. Presentación y aplicación del modelo del doble logaritmo de la función de Gompertz

a) Presentación de la información

Las tasas específicas de fecundidad que se ajustaron, fueron tomadas de la Encuesta Mexicana de Fecundidad; 4 dichas tasas corresponden a los años 1973, 1974 y 1975 (nivel nacional). El modelo que se aplicará requiere una estructura de la fecundidad por edades estándar, por ello, se toma como tal a la estructura del año de 1975, sin que esto quiera decir que es el mejor o el único estándar; sólo es un estándar tomado para ejemplificar el uso del modelo.

En el cuadro 1 se dan los valores de las tasas específicas de fecundidad, correspondientes a cada año.

Cuadro 1

MÉXICO: T ASAS ESPECÍFICAS DE FECUNDIDAD, 1973, 74 y 75

\begin{tabular}{cccc}
\hline \multirow{2}{*}{$\begin{array}{c}\text { Grupo de } \\
\text { edades }\end{array}$} & \multicolumn{3}{c}{ Tasas especificas de fecundidad } \\
\cline { 2 - 4 } & $5 \mathrm{fx}$ & $5 \mathrm{fx}$ & $5 \mathrm{fx}$ \\
& 1973 & 1974 & 1975 \\
\hline $15-19$ & 0.1092 & 0.1061 & 0.0989 \\
$20-24$ & 0.2870 & 0.2872 & 0.2964 \\
$25-29$ & 0.3300 & 0.2805 & 0.2941 \\
$30-34$ & 0.2702 & 0.2624 & 0.2359 \\
$35-39$ & 0.1868 & 0.1645 & 0.1840 \\
$40-44$ & 0.0887 & 0.0820 & 0.0793 \\
$45-49$ & 0.0175 & 0.0172 & 0.0165 \\
\hline
\end{tabular}

Fuente: Volumen II del primer informe nacional de los resultados de la Encuesta Mexicana de Fecundidad, cuadro H.E. 1 y H.E. 2

Se calcula, para cada año, la tasa global de fecundidad (como la suma de las tasas específicas multiplicadas por cinco). En el cuadro 2 se dan los valores de las tasas globales de fecundidad (TGF) para cada año.

b) Presentación y aplicación del modelo de J. Chackiel para ajustar la estructura por edad de la fecundidad.

4 Secretaría de Programación y Presupuesto; Encuesta Mexicana de Fecundidad, primer informe nacional, Volumen I, México, febrero 1979, pp. 135-173. 
Cuadro 2

MÉXICO: TASAS GLOBALES DE FECUNDIDAD 1973, 74 y 75

\begin{tabular}{lc}
\hline año & T G F \\
\hline 1973 & 6.45 \\
1974 & 6.00 \\
1975 & 6.03 \\
\hline
\end{tabular}

La representación de la fecundidad acumulada mediante la función de Gompertz sería la siguiente:

$$
F(x)=(T G F) A^{B^{X}}
$$

donde:

x - es la variable edad

$F(x)$ - es la fecundidad acumulada hasta la edad x (vía suma de las tasas).

(TFG) - Tasa global de fecundidad que es el valor de $F(x)$ para la edad límite superior del período reproductivo.

A y B - Son parámetros que varían alrededor de 0 y 1 respectivamente.

La distribución relativa acumulada adopta entonces la siguiente forma:

$$
\frac{F(x)}{(T G F)}=A^{B^{X}}
$$

Aplicando logaritmo natural a (2) se tiene:

$$
\ln \frac{F(x)}{T G F}=B^{x} \ln A
$$


Dado que los miembros de la igualdad son negativos, se multiplica por $(-1)$ y se le aplica nuevamente logaritmo natural:

$$
\ln \left[-\ln \frac{F(x)}{T G F}\right]=x \ln B+\ln (-\ln A)
$$

lo que constituye una recta de la forma:

$$
V(x)=a_{0}+b_{0} x
$$

en donde:

$$
\begin{aligned}
& V(x)=\ln \left[-\ln \frac{F(x)}{T G F}\right] \\
& a_{0}=\ln (-\ln A) \\
& b_{0}=\ln B
\end{aligned}
$$
caso:

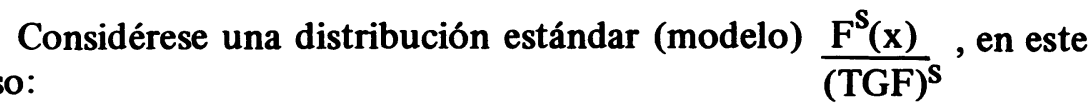

$$
\mathbf{v}^{\mathbf{s}}(\mathbf{x})=\mathbf{a}_{\mathbf{s}}+\mathbf{b}_{\mathbf{s}} \mathbf{x}
$$

en donde:

$$
V^{S}(x)=\ln \left[-\ln \frac{F^{s}(x)}{(T G F)^{S}}\right]
$$

Si $V(x)$ y $V^{\mathbf{s}}(x)$ se comportan linealmente con respecto a la edad $x$, tamuién habrá una relación lineal entre ellos, ya que de $(9)$ se obtiene:

$$
x=\frac{1}{b_{s}} v^{s}(x)-\frac{a_{s}}{b_{s}}
$$

que sustituyendo en (5):

$$
V(x)=a_{0}-a_{s} \frac{b_{0}}{b_{s}}+\frac{b_{0}}{b_{s}} V^{s}(x)
$$


y iando:

$$
a=a_{0}-a_{s} \frac{b_{0}}{b_{s}} y \quad b=\frac{b_{0}}{b_{s}}
$$

se tiene que:

$$
\mathrm{V}(\mathrm{x})=\mathrm{a}+\mathrm{bV}^{\mathrm{s}}(\mathrm{x})
$$

En el cuadro 3 se aprecia el procèso de cálculo de las funciones $\mathrm{V}(\mathrm{x})$ y $\mathrm{V}^{\mathrm{S}}(\mathrm{x})$, para los años 1973, 1974 y 1975.

\section{Cuadro 3}

MÉXICO: CÁlCULO DEL DOBLE LOGARITMO DE LA ESTRUCTURA

\begin{tabular}{|c|c|c|c|}
\hline $\begin{array}{l}\text { Grupo de } \\
\text { edades }\end{array}$ & $5 f x$ & $\frac{F(x)}{T G F}$ & $V(x)$ \\
\hline & & año 1973 & \\
\hline $\begin{array}{l}15-19 \\
20-24 \\
25-29 \\
30-34 \\
35-39 \\
40-44 \\
45-49\end{array}$ & $\begin{array}{l}0.1092 \\
0.2870 \\
0.3300 \\
0.2702 \\
0.1868 \\
0.0887 \\
0.0175\end{array}$ & $\begin{array}{c}-\overline{-} \\
0.0847 \\
0.3073 \\
0.5632 \\
0.7728 \\
0.9176 \\
0.9864\end{array}$ & $\begin{array}{r}-- \\
0.9037 \\
0.1655 \\
-0.5549 \\
-1.3556 \\
-2.4539 \\
-4.2929\end{array}$ \\
\hline \multirow[t]{2}{*}{ TGF } & 6.45 & & \\
\hline & & año 1974 & \\
\hline $\begin{array}{l}15-19 \\
20-24 \\
25-29 \\
30-34 \\
35-39 \\
40-44 \\
45-49\end{array}$ & $\begin{array}{l}0.1061 \\
0.2872 \\
0.2805 \\
0.2624 \\
0.1645 \\
0.0820 \\
0.0172\end{array}$ & $\begin{array}{c}-\overline{0} \\
0.0884 \\
0.3278 \\
0.5615 \\
0.7802 \\
0.9173 \\
0.9857\end{array}$ & $\begin{array}{r}-- \\
0.8861 \\
0.1092 \\
-0.5498 \\
-1.3937 \\
-2.4500 \\
-4.2379\end{array}$ \\
\hline \multirow[t]{2}{*}{ TGF } & 6.00 & & \\
\hline & & año 1975 & \\
\hline $\begin{array}{l}15-19 \\
20-24 \\
25-29 \\
30-34 \\
35-39 \\
40-44 \\
45-49\end{array}$ & $\begin{array}{l}0.0989 \\
0.2964 \\
0.2941 \\
0.2359 \\
0.1840 \\
0.0793 \\
0.0165\end{array}$ & $\begin{array}{c}-- \\
0.0821 \\
0.3280 \\
0.5721 \\
0.7678 \\
0.9205 \\
0.9863\end{array}$ & $\begin{array}{r}-- \\
0.9164 \\
0.1086 \\
-0.5825 \\
-1.3310 \\
-2.4909 \\
-4.2841\end{array}$ \\
\hline TGF & 6.03 & & \\
\hline
\end{tabular}
DE FECUNDIDAD ACUMULADA. 1973, 1974 y 1975

Los valores de a y b se obtienen ajustando los 6 puntos (que se obtienen al graficar los valores de $V^{\mathbf{S}}(\mathrm{x})$, en el eje de las abscisas, $y$ $\mathrm{V}(\mathrm{x})$, en el eje de las ordenadas (ver gráficas 1 y 2 ) por el método de promedios, ${ }^{5}$ en que se plantean las siguientes ecuaciones.

5 Ver: Brass, W.: Métodos para estimar la fecundidad y la mortalidad en poblaciones con datos limitados. CELADE, Serie E, No. 14, página 135. 


\section{Gráfica 1}

MÉXICO: DOBLE LOGARITMO DE LA ESTRUCTURA DE LA FECUNDIDAD ACUMULADA DEL AÑO 1973, CON RESPECTO AL AÑO 1975 CONSIDERADO COMO ESTÁNDAR

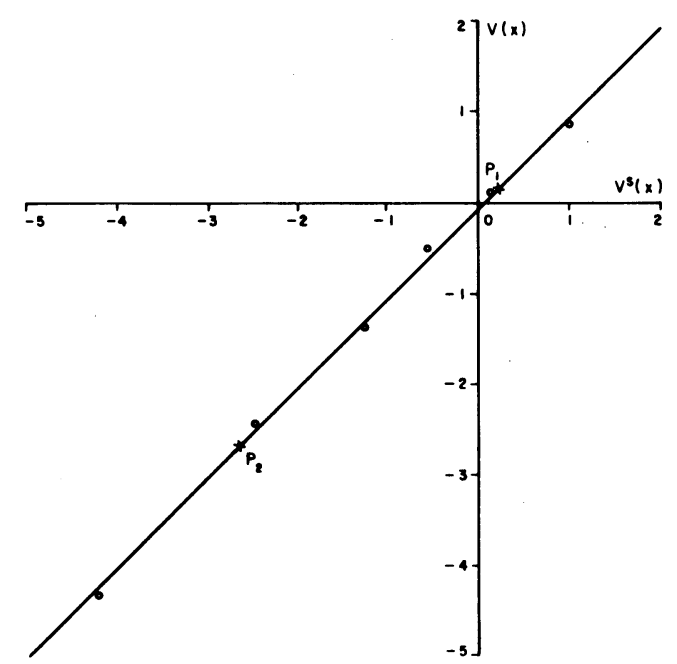

Gráfica 2

MÉXICO: DOBLE LOGARITMO DE LA ESTRUCTURA DE LA FECUNDIDAD ACUMULADA DEL AÑO 1974, CON RESPECTO AL AÑO 1975 CONSIDERADO COMO ESTÁNDAR

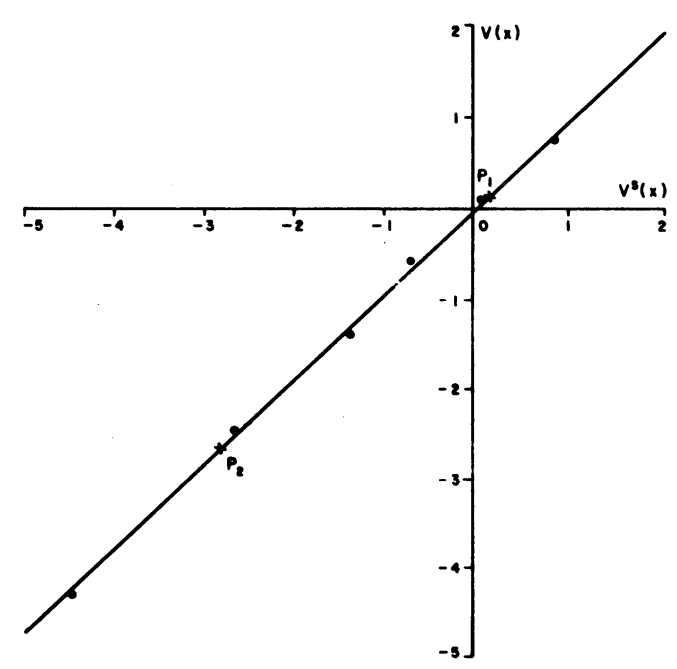


MINA: ESTRUCTURA DE LA FECUNDIDAD

$$
\begin{aligned}
& \bar{V}_{1}(x)=a+b \bar{V}_{1}^{s}(x) \\
& \bar{V}_{2}(x)=a+b \bar{V}_{2}^{s}(x)
\end{aligned}
$$

donde:

$$
\begin{aligned}
& \overline{\mathrm{V}}_{1}=\frac{\mathrm{V}(20)+\mathrm{V}(25)+\mathrm{V}(30)}{3} \quad \mathrm{y} \\
& \overline{\mathrm{V}}_{2}=\frac{\mathrm{V}(35)+\mathrm{V}(40)+\mathrm{V}(45)}{3} \\
& \overline{\mathrm{V}}_{1}^{\text {s }} \text { y } \overline{\mathrm{V}}_{2}^{\text {s }} \quad \begin{array}{l}
\text { son los mismos conceptos para la distribución } \\
\text { estándar. }
\end{array}
\end{aligned}
$$

En el cuadro 4 se resume el procedimiento anterior con los datos provenientes de la Encuesta.

\section{Cuadro 4}

PUNTOS MEDIOS OBTENIDOS A PARTIR DEL MÉTODO DE PROMEDIOS

\begin{tabular}{lc}
\hline año & puntos medios \\
\hline \multirow{2}{*}{1973} & $\mathrm{P}_{1}=\left(\overline{\mathrm{v}}_{1}, \overline{\mathrm{v}}_{1}^{\mathrm{s}}\right)=(0.1475,0.1714)$ \\
& $\mathrm{P}_{2}=\left(\overline{\mathrm{v}}_{2}, \overline{\mathrm{v}}_{2}^{\mathrm{s}}\right)=(-2.7020,-2.7008)$ \\
1974 & $\mathrm{P}_{1}=\left(\overline{\mathrm{V}}_{1}, \overline{\mathrm{v}}_{1}^{\mathrm{s}}\right)=(0.1475,0.1485)$ \\
& $\mathrm{P}_{2}=\left(\overline{\mathrm{v}}_{2}, \overline{\mathrm{v}}_{2}^{\mathrm{s}}\right)=(-2.7020,-2.6939)$ \\
\hline
\end{tabular}

A partir de los datos del cuadro 4 obtenemos los valores de a y b, los cuales nos servirán para ajustar las estructuras de la fecundidad por edades.

Antes de presentar los ajustes se da a continuación una breve explicación del significado de los parámetros a y b. 
a y b son los parámetros que diferencian la estructura observada del estándar. El parámetro a representa la diferencia en la edad media de la fecundidad y b está ligado con la dispersión de las tasas de fecundidad por edades.

Tomando $\mathrm{b}=1$ constante $\mathrm{y}$ variando $\mathrm{a}$, se observa que un $\mathrm{a}<0$ traslada la curva hacia la izquierda, señalando una menor edad media de la fecundidad, mientrasuna a $>0$ conduce a lo contrario (ver gráfica 3).

Si se mantiene constante $a=0$ y se varía el valor de $b$, se observa que una $b>1$ significa mayor concentración de las tasas con respecto al estándar y una $\mathrm{b}<1$ representa una menor concentración (ver gráfica 4).

- En los cuadros 5 y 6 se resume el procedimiento para la obtención de los objetos.

\section{Cuadro 5}

MÉXICO: AJUSTE DE LA ESTRUCTURA DE LA FECUNDIDAD POR EDADES 1973

\begin{tabular}{|c|c|c|c|c|c|c|}
\hline $\begin{array}{l}\text { Grupo de } \\
\text { edad }\end{array}$ & $\begin{array}{l}\text { Estructura } \\
\text { observada }\end{array}$ & $v(x)$ & $v^{8}(x)$ & $\hat{v}(x)$ & $\frac{\hat{F}(x)}{T G F}$ & $\begin{array}{l}\text { Estructura } \\
\text { ajustada }\end{array}$ \\
\hline $15-19$ & 0.1092 & -- & -- & -- & -- & 0.0980 \\
\hline $20-24$ & 0.2870 & 0.9036 & 0.9164 & 0.9464 & 0.0760 & 0.3138 \\
\hline $25-29$ & 0.3300 & 0.1655 & 0.1086 & 0.1322 & 0.3194 & 0.3184 \\
\hline $30-34$ & 0.2702 & -0.5549 & -0.5825 & -0.5644 & 0.5663 & 0.2566 \\
\hline $35-39$ & 0.1868 & -1.3556 & -1.3610 & -1.3189 & 0.7653 & 0.199 .9 \\
\hline $40-44$ & 0.0887 & -2.4539 & -2.4909 & -2.4880 & 0.9203 & 0.0854 \\
\hline \multirow[t]{2}{*}{$45-49$} & 0.0175 & -4.2929 & -4.2841 & -4.2955 & 0.9865 & 0.0174 \\
\hline & \multicolumn{2}{|c|}{$a=0.0227$} & $\mathbf{b}=$ & & $=a+$ & x) \\
\hline
\end{tabular}

Cuadro 6

MÉXICO: AJUSTE DE LA ESTRUCTURA DE LA FECUNDIDAD POR EDADES 1974

\begin{tabular}{ccccccc}
\hline $\begin{array}{c}\text { Grupo de } \\
\text { edad }\end{array}$ & $\begin{array}{c}\text { Estructura } \\
\text { observada }\end{array}$ & $v(x)$ & $v^{*}(x)$ & $\hat{v}(x)$ & $\frac{\hat{F}(x)}{\text { TGF }}$ & $\begin{array}{c}\text { Estructura } \\
\text { ajustada }\end{array}$ \\
\hline $15-19$ & 0.1061 & -- & -- & -- & -- & 0.0988 \\
$20-24$ & 0.2872 & 0.8861 & 0.9164 & 0.9155 & 0.0823 & 0.2943 \\
$25-29$ & 0.2805 & 0.1092 & 0.1086 & 0.1097 & 0.3276 & 0.2923 \\
$30-34$ & 0.2624 & -0.5498 & -0.5825 & -0.5797 & 0.5712 & 0.2348 \\
$35-39$ & 0.1645 & -1.3937 & -1.3937 & -1.3263 & 0.7669 & 0.1836 \\
$10-44$ & 0.0820 & -2.4500 & -2.4909 & -2.4833 & 0.9199 & 0.0794 \\
$45-49$ & 0.0172 & -4.2379 & -4.2841 & -4.2721 & 0.9861 & 0.0167 \\
& $a=0.0014$ & $b=0.09975$ & $\hat{v}(x)=a+b$ v $(x)$ \\
\hline
\end{tabular}

Observación: A continuación se describe el procedimiento de ajuste de esta información mediante las ideas desarrolladas anteriormente. 
Gráfica 3

MODIFICACIÓN DE LA ESTRUCTURA DE LA FECUNDIDAD TOMANDO $b=1$ Y HACIENDO VARIAR a MEDIANTE LA RELACIÓN $v(x)=a+b v^{s}(x)$

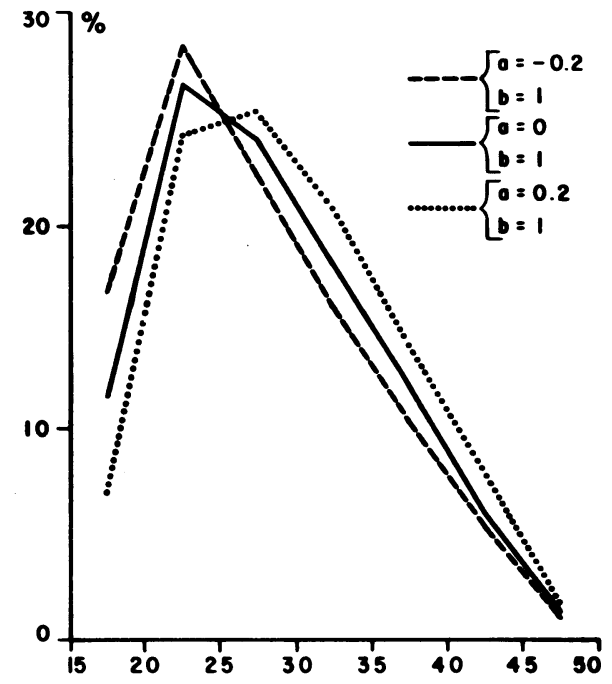

Fuente: Chackicl, J. , op. cit., pág. 19.

Gráfica 4

MODIFICACIONES DE LA ESTRUCTURA DE LA FECUNDIDAD TOMANDO $a=0$ HACIENDO VARIAR b MEDIANTE LA RELACIÓN $V(x)=a+b V^{s}(x)$

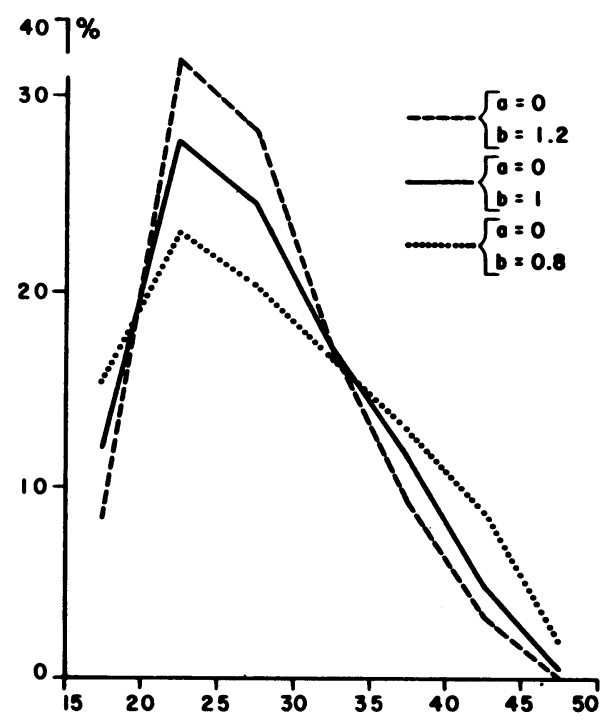

Fuente: Chackiel, J., op. cit., pág. 20. 
36

DEMOGRAFIA Y ECONOMIA XVI: 1,1982

Dada una estructura estándar $V^{\mathbf{S}}(\mathrm{x})$ y estimados los parámetros a y $b$ se está en condiciones de obtener los valores $V(x)$ ajustados mediante la relación:

$$
\hat{V}(x)=a+b V^{s}(x)
$$

y luego:

$$
\frac{F(x)}{T G F}=e^{-e^{\hat{v}}(x)}
$$

que desacumulando conduce a la estructura ajustada.

Finalmente, en las gráficas 5 y 6 se presentan los ajustes obtenidos al aplicar el modelo del doble logaritmo de la función de Gompertz. Nótese que para el caso de la estructura de la fecundidad del año 1974, el ajuste que se obtiene es más adecuado que el obtenido para 1973, lo cual se debe al estándar que se emplea -estructura de la fecundidad del año 1975 - el cual se asemeja más al del año 1974 que al de 1973.

Gráfica 5

MÉXICO: ESTRUCTURA DE LA FECUNDIDAD OBSERVADA Y AJUSTADA, 1973

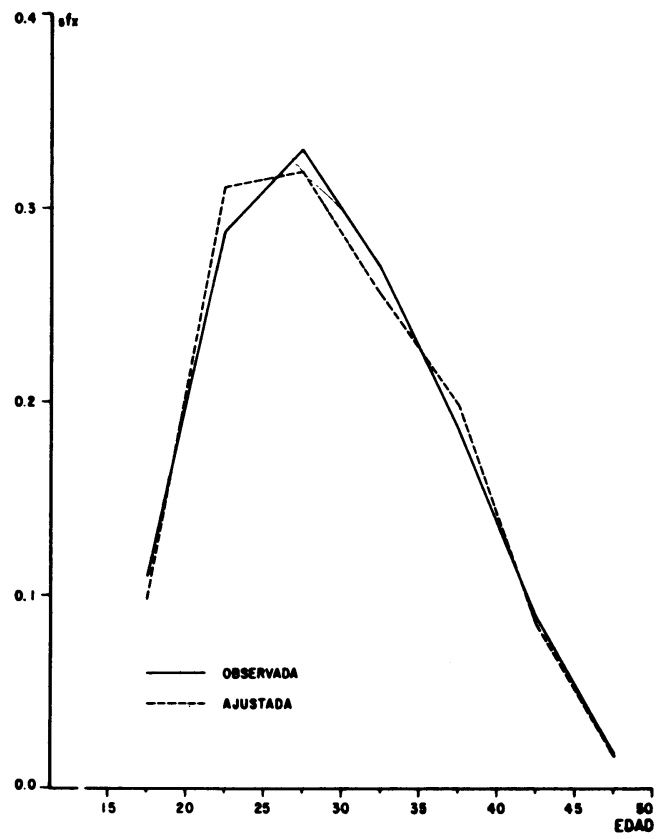


Gráfica 6

MÉXICO: ESTRUCTURA DE LA FECUNDIDAD OBSERVADA Y AJUSTADA, 1974

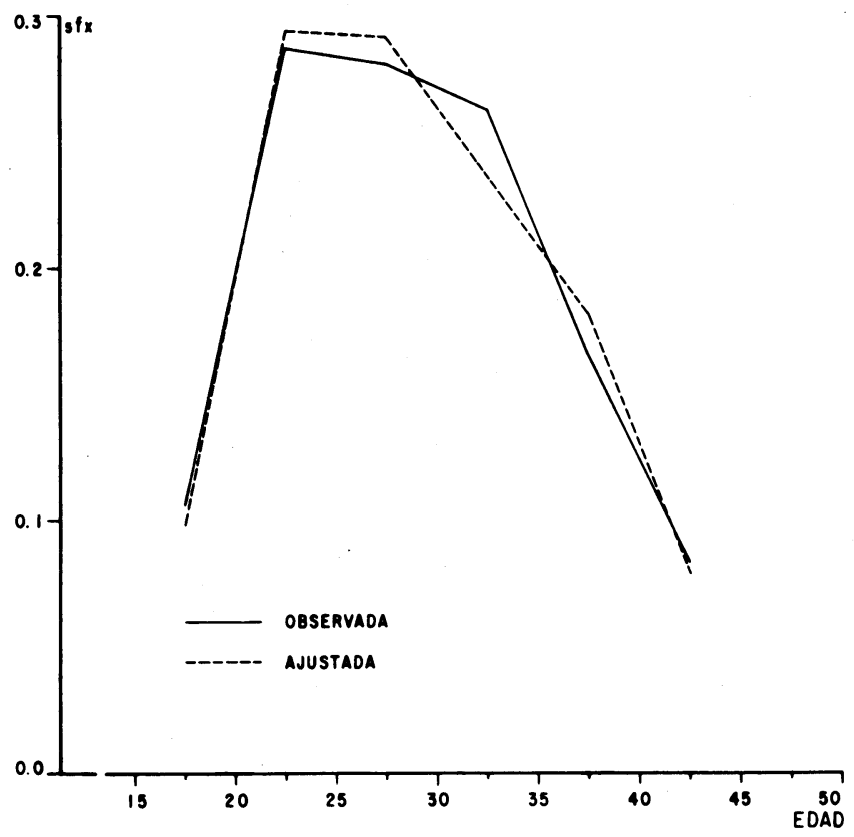

Cabe señalar que los valores de los parámetros a y b para la estructura de la fecundidad del año 1973 (0.0227 y 1.0080 respectivamente) denotan por un lado, que existe en 1973 una edad media de la fecundidad mayor a la de la estructura estándar de 1975 (29.33 años contra 29.22 años) y por otro lado, que hay una mayor concentración en las tasas en 1973 con respecto al estándar. Lo contrario ocurre para el caso del ajuste de la estructura por edad de la fecundidad de 1974 , ya que para dicho caso los valores de a y b son: 0.0014 y 0.9975 , respectivamente, lo que indica que existe en 1974 una edad media de la fecundidad mayor a la de la estructura estándar de 1975 (29.23 años contra 29.22 años) y una mayor concentración en las tasas.

c) Desagregación de la estructura por edad de la fecundidad

Obtenido el ajuste de las tasas específicas de fecundidad, se puede emṕlear el modelo del doble logaritmo de la función de Gompertz, 
para desagregar en edades individuales la estructura por edad de la fecundidad, la cual se tiene para grupos de edad quinquenales.

El procedimiento para desagregar la estructura de la fecundidad, se ilustra tomando la estructura corregida para el año 1973, la cual se muestra en el cuadro 5; en él aparecen los valores $\hat{V}(x)$ para $x=20$, $25,30,35,40$ y 45 . Graficando los valores $\hat{V}(x)$ se observa la tendencia lineal de ellos (ver gráfica 7) la cual era de esperarse debido a que:

De (4):

$$
\ln \left[-\ln \frac{F(x)}{T G F}\right]=x \ln B+\ln (-\ln A)
$$

la cual es una recta de la forma vista en (5):

$$
\hat{V}(x)=a_{0}+b_{o} x
$$

Los valores de $a_{o}$ y $b_{o}$ se obtienen de la misma forma en que se obtuvieron los valores a y $b\left[V(x)=a+b V^{s}(x)\right]$, es decir, ajustando los 6 puntos $\hat{\mathrm{V}}(\mathrm{x})$ por el método de promedios.

En este caso las ecuaciones que se plantean son:

$$
\begin{aligned}
& \hat{\bar{V}}_{1}(x)=a_{o}+b_{o} \bar{X}_{1} \\
& \hat{\bar{V}}_{2}(x)=a_{o}+b_{o} \bar{X}_{2}
\end{aligned}
$$

donde:

$$
\begin{aligned}
& \hat{\bar{V}}_{1}=\frac{V(20)+V(25)+V(30)}{3} \\
& \hat{\bar{V}}_{2}=\frac{V(35)+V(40)+V(45)}{3} \\
& \bar{X}_{1}=\frac{20+25+30}{3}=25 \\
& \bar{X}_{2}=\frac{35+40+45}{3}=40
\end{aligned}
$$


Gráfica 7

MÉXICO: DISTRIBUCIÓN DE LOS VALORES V̂(x), 1973

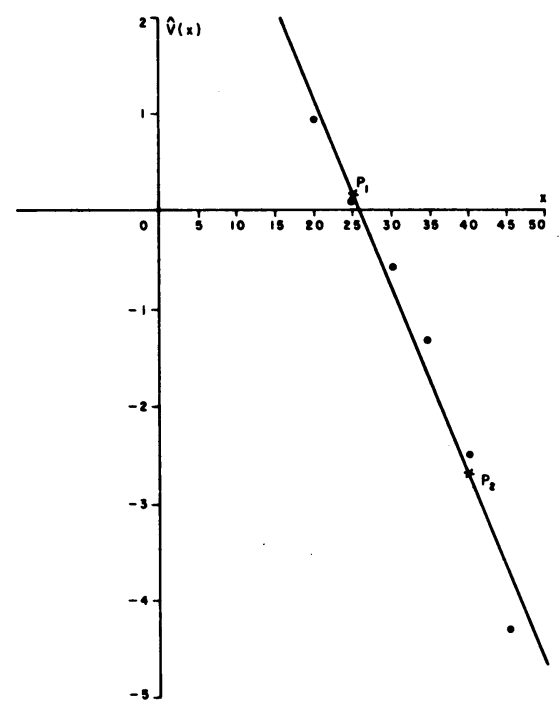

Para los valores $\hat{V}(x)$ del año 1973 se tiene:

$\hat{\overline{\mathrm{V}}}_{1}=0.1714 \quad ; \quad \hat{\overline{\mathrm{V}}}_{2}=-2.7008$

Con los dos puntos, $P_{1}=(25,0.1608)$ y $P_{2}(40,-2.6945)$, se obtienen los valores de $a_{0} y b_{0}$, estimando la recta que pasa por esos puntos, la cual es:

$$
\hat{\mathrm{V}}(\mathrm{x})-\hat{\overline{\mathrm{V}}}_{1}=\frac{\hat{\overline{\mathrm{V}}}_{2}-\hat{\overline{\mathrm{V}}}_{1}}{\overline{\mathrm{X}}_{2}-\overline{\mathrm{X}}_{1}}\left(\mathrm{X}-\overline{\mathrm{X}}_{1}\right)
$$

Para los valores $\hat{V}(x)$ de 1973 se tiene:

$$
\hat{V}(x)=4.95840-0.19148 x
$$

donde: $\quad a_{0}=4.95840 \quad$ y $\quad b_{0}=-0.19148$

Evaluando la ecuación 28 para $x=10,11,12, \ldots, 50$ se obtienen los valores $\hat{V}(x)$ para dichas edades y empleando (18) se estiman los valores $\hat{F}(x) / T G F$ y conocida la tasa global de fecundidad (6.45 
para 1973) se calculan los valores $\hat{F}(x)$ para esas edades. En el cuadro 7 se dan los valores obtenidos para el año 1973.

\section{Cuadro 7}

MÉXICO: VALORES DE V̂ (x), $\hat{\mathrm{F}}$ (x)/TGF Y F̂ (x), POR EDADES INDIVIDUALES, 1973

\begin{tabular}{|c|c|c|c|}
\hline $\mathbf{x}$ & $\hat{\mathrm{v}}(\mathrm{x})$ & $\frac{\hat{F}(x)}{T G F}$ & $\hat{\mathrm{F}}(\mathrm{x})$ \\
\hline $\begin{array}{l}10 \\
11 \\
12 \\
13 \\
14 \\
15 \\
16 \\
17 \\
18 \\
19 \\
20 \\
21 \\
22 \\
23 \\
24 \\
25 \\
26 \\
27 \\
28 \\
29 \\
30 \\
31 \\
32 \\
33 \\
34 \\
35 \\
36 \\
37 \\
38 \\
39 \\
40 \\
41 \\
42 \\
43 \\
44 \\
45 \\
46 \\
47 \\
48 \\
49 \\
50\end{array}$ & $\begin{array}{l}3.0436 \\
2.8521 \\
2.6606 \\
2.4692 \\
2.2777 \\
2.0862 \\
1.8947 \\
1.7032 \\
1.5118 \\
1.3203 \\
1.1288 \\
0.9373 \\
0.7458 \\
0.5544 \\
0.3629 \\
0.1714 \\
-0.0201 \\
-0.2116 \\
-0.4030 \\
-0.5945 \\
-0.7860 \\
-0.9775 \\
-1.1690 \\
-1.3604 \\
-1.5519 \\
-1.7434 \\
-1.9349 \\
-2.1264 \\
-2.3178 \\
-2.5093 \\
-2.7008 \\
-2.8923 \\
-3.0838 \\
-3.2752 \\
-3.4667 \\
-3.6582 \\
-3.8497 \\
-4.0412 \\
-4.2326 \\
-4.4241 \\
-4.6156\end{array}$ & $\begin{array}{l}0.0000000008 \\
0.0000000299 \\
0.0000006130 \\
0.0000074077 \\
0.0000580492 \\
0.0003177484 \\
0.0012933068 \\
0.0041216897 \\
0.0107281275 \\
0.0236463958 \\
0.0454135872 \\
0.0778416385 \\
0.1214652261 \\
0.1753631813 \\
0.2375226948 \\
0.3051458438 \\
0.3752733226 \\
0.4451747630 \\
0.5125730204 \\
0.5758901109 \\
0.6340269548 \\
0.6864303261 \\
0.7329502410 \\
0.7737100150 \\
0.8090900276 \\
0.8395202808 \\
0.8655079561 \\
0.8875717826 \\
0.9062047315 \\
0.9218938917 \\
0.9350532777 \\
0.9460607122 \\
0.9552473729 \\
0.9628965414 \\
0.9692624219 \\
0.9745505653 \\
0.9789388292 \\
0.9825771952 \\
0.9855902256 \\
0.9880865410 \\
0.9901525628\end{array}$ & $\begin{array}{l}0.0000000052 \\
0.0000001929 \\
0.0000039539 \\
0.0000477797 \\
0.0003744173 \\
0.0020494772 \\
0.0083418289 \\
0.0265848986 \\
0.0691964224 \\
0.1525192529 \\
0.2929176374 \\
0.5020785683 \\
0.7834507083 \\
1.1310925190 \\
1.5320213810 \\
1.9681906930 \\
2.4205129310 \\
2.8713772210 \\
3.3060959820 \\
3.7144912150 \\
4.0894738580 \\
4.4274756030 \\
4.7275290540 \\
4.9904295970 \\
5.2180678020 \\
5.4149058110 \\
5.5825263170 \\
5.7248379980 \\
5.8450205180 \\
5.9462156010 \\
6.0310936410 \\
6.1020915940 \\
6.1613455550 \\
6.2106826920 \\
6.2517426210 \\
6.2858511460 \\
6.3141554480 \\
6.3376229090 \\
6.3570569550 \\
6.3731581890 \\
6.3864840300\end{array}$ \\
\hline
\end{tabular}

Finalmente, para obtener la estructura por edad de la fecundidad, por edades individuales, se desagrega la función $\hat{F}(x)$ ya que:

$$
\hat{\mathbf{f}}(\mathbf{x})=\hat{\mathbf{F}}(\mathrm{x}+1)-\mathbf{F}(\mathbf{x})
$$

En el cuadro 8 se dan los valores de las tasas específicas de fecundidad, por edad individual, y en la gráfica 8 la distribución de dichas tasas. 
Cuadro 8

MÉXICO: TASAS ESPECÍFICAS DE FECUNDIDAD, POR EDADES

INDIVIDUALES, 1973

\begin{tabular}{cccc}
\hline$\underline{x}$ & $f(\underset{x}{\mathbf{x}})$ & $\underline{x}$ & $f(\underline{x})$ \\
\hline 10 & 0.0000001877 & 30 & 0.3380017450 \\
11 & 0.0000037610 & 31 & 0.3000534510 \\
12 & 0.0000438258 & 32 & 0.2629005430 \\
13 & 0.0003266376 & 33 & 0.2276382050 \\
14 & 0.0016750599 & 34 & 0.1968380090 \\
15 & 0.0083418289 & 35 & 0.1676205060 \\
16 & 0.0182430697 & 36 & 0.1423116810 \\
17 & 0.0426115238 & 37 & 0.1201825200 \\
18 & 0.0833228305 & 38 & 0.1011904210 \\
19 & 0.1403983845 & 39 & 0.0848780400 \\
20 & 0.2091609309 & 40 & 0.0709979530 \\
21 & 0.2813721400 & 41 & 0.0592539610 \\
22 & 0.3476418107 & 42 & 0.0493371370 \\
23 & 0.4009288620 & 43 & 0.0410599290 \\
24 & 0.4361693120 & 44 & 0.0341085250 \\
25 & 0.4523222380 & 45 & 0.0283043020 \\
26 & 0.4508642900 & 46 & 0.0234674610 \\
27 & 0.4347187610 & 47 & 0.0194340460 \\
28 & 0.4083952330 & 48 & 0.0161012340 \\
29 & 0.3749826430 & 49 & 0.0133258410 \\
\hline
\end{tabular}

A manera de conclusión se puede decir que el modelo del doble logaritmo de la función de Gompertz sirve para realizar ajustes en la estructura por edad de la fecundidad y que el éxito que se tenga al aplicarlo depende de la adecuada estructura por edad estándar utilizada. Para el caso de México queda por encontrar el estándar adecuado (en este trabajo se tomó como estructura por edad estándar la del año de 1975, únicamente para ejemplificar el uso del modelo).

Otra de las ventajas del modelo, es la de poder desagregar la estructura por edad de la fecundidad (la cual se tiene en tasas específicas de fecundidad por grupos quinquenales de edades), en edades individuales.

Cabe señalar que otro uso del modelo es para proyectar la estructura por edad de la fecundidad, observando la tendencia de los parámetros a y $b$, de la expresión $\hat{V}(x)=a+b V^{S}(x)$ a través del tiempo, o bien los parámetres $a_{n}$ y $b_{0}$ de la expresión $V(x)=a_{0}+b_{0} x$, también en diferentes mo.: entos. 


\section{Gráfica 8}

MÉXICO: DISTRIBUCIÓN, POR EDADES INDIVIDUALES, DE L ‘ TASAS

ESPECÍFICAS DE FECUNDILAD, 1973

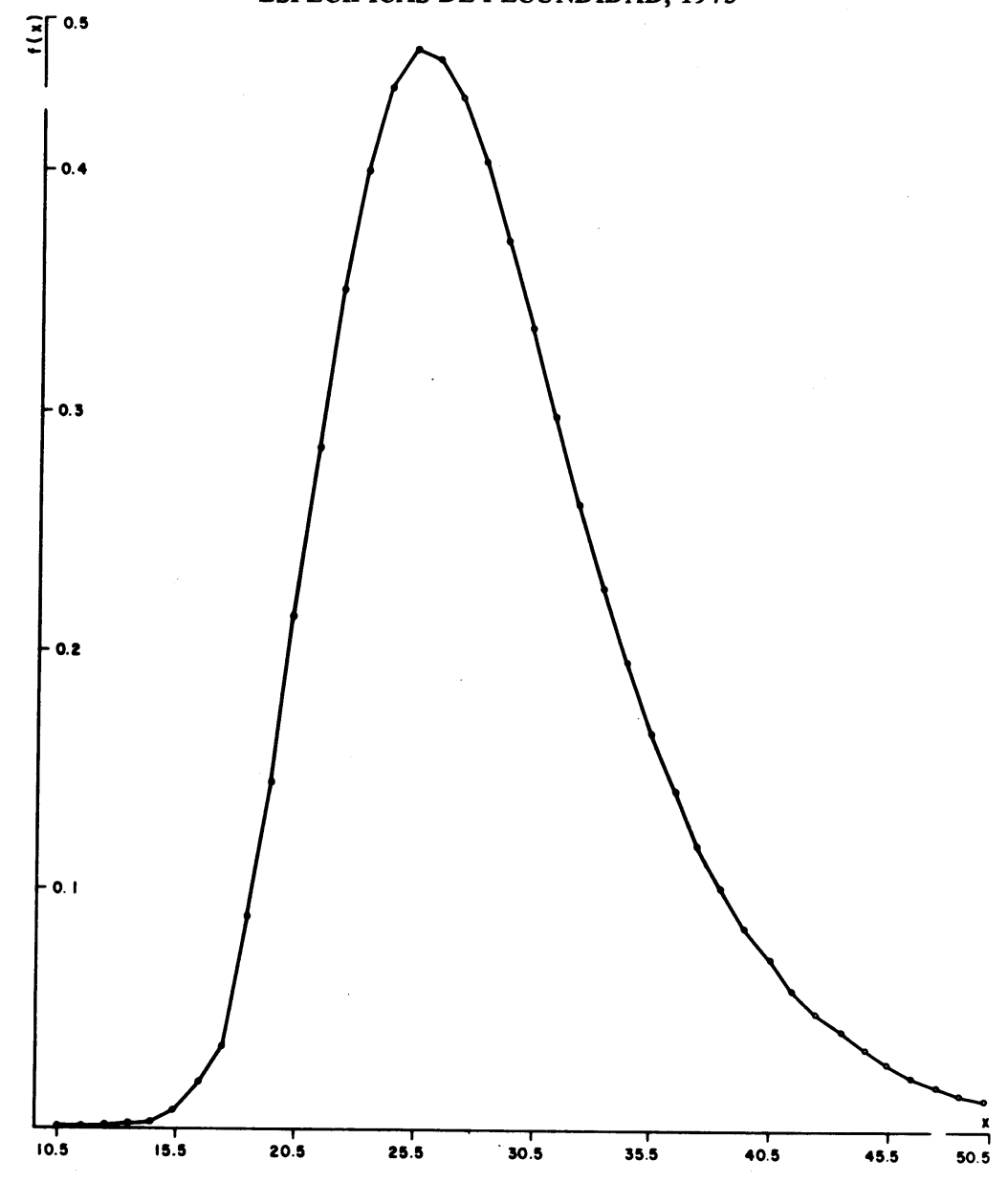

ANEXO

Uno de los esfuerzos hechos para dar una ley matemática que describa la mortalidad experimentada por una población dada, fue el hecho por Gompertz. La ley que desarrolló (Ley de Gompertz) supone que la resistencia del hombre a la muerte decrece en una tasa proporcional a sí misma.

Si se denota como $M_{X}$ (tasa instantánea de mortalidad) a la sus- 
ceptibilidad del hombre a la muerte, y al recíproco de ella, $\frac{1}{\mathrm{M}_{\mathrm{x}}}$, como la resistencia del hombre a la muerte, entonces, el supuesto de la ley de Gompertz se puede expresar de la siguiente manera:

$$
\frac{\mathrm{d}}{\mathrm{d}_{\mathrm{X}}}\left(1 / \mathrm{M}_{\mathrm{X}}\right)=-\mathrm{h}\left(\frac{1}{\mathrm{M}_{\mathrm{X}}}\right)
$$

donde $h$ es la tasa proporcional a la cual decrece la resistencia del hombre a la muerte.

Desarrollando (1), obtenemos:

$$
\begin{aligned}
& \int \frac{d\left(1 / M_{X}\right)}{1 / M_{X}}=-h \int d_{X} \\
& \ln \left(1 / M_{X}\right)+l_{n} B=-h_{X} \\
& \ln \frac{B}{M_{X}}=-h_{X} \\
& { }_{M_{X}}^{B}=e^{-h_{X}} \\
& M_{X}=B e^{h_{x}}
\end{aligned}
$$

Se denota a $\mathrm{e}^{\mathrm{h}}$ como $\mathrm{C}$, entonces (6) se puede expresar como:

$$
\mathrm{M}_{\mathbf{X}}=\mathrm{BC}^{\mathbf{X}}
$$

La tasa instantánea de mortalidad $\mathbf{M}_{\mathbf{X}}$ se define como:

$$
\begin{aligned}
M_{X} & =\frac{l_{i m}}{h \rightarrow 0} \frac{l_{(x)}-l_{(x+h)}}{h l(x)} \\
& =\frac{-1}{1(x)} \lim _{h \rightarrow 0} \frac{1(x+h)-1(x)}{h} \\
& =-\frac{1}{1(x)} \frac{d}{d_{x}} 1(x) \\
M_{x} & =-\frac{d}{d_{x}} 1_{n} l_{x}
\end{aligned}
$$


Sustituyendo (11) en (7) se obtiene:

$$
\begin{aligned}
& -\frac{d}{d_{x}} 1_{n} 1_{x}=B C^{x} \\
& \int \frac{d}{d_{x}} 1_{n} 1_{x} d_{x}=-\int B C^{x} d_{x} \\
& \int \frac{d}{d_{x}} 1_{n} 1_{x} d_{x}=-\int \frac{B}{l_{n} C} C^{x} 1_{n} C d_{x} \\
& l_{n} 1_{x}=-\frac{B C^{x}}{1_{n} C}+1_{n} K
\end{aligned}
$$

se denota a $-\frac{B}{l_{n} C}$ como $1_{n} g$, entonces (15) se puede expresar como:

$$
\begin{aligned}
1_{n} 1_{x} & =C^{x} 1_{n} g+1_{n} K \\
1_{n} 1_{x} & =1_{n} K_{g} C^{x} \\
1_{x} & =K g^{C^{x}}
\end{aligned}
$$

Cuando se habla de la función de Gompertz se entiende como tal a:

$$
\mathrm{Y}(\mathrm{x})=\mathrm{Kg}^{\mathrm{C}^{\mathrm{x}}}
$$

En el caso de la aplicación de la función de Gompertz al fenómeno fecundidad, se tiene que (19) toma la expresión:

$$
F(x)=(T G F) A^{B^{x}}
$$




\section{BIBLIOGRAFIA}

Brass, W., The relational Gompertz model of fertility by age of woman. (Inédito).

Brass, W., An application of the relational Gompertz model of fertility. (Inédito).

Camisa, Z., Introducción al estudio de la fecundidad. CELADE, Serie B, No. 1007, San José, Costa Rica, 1975.

Carrasco, E., Modelos matemáticos para ajustar curvas acumulativas de fecundidad. CELADE, Serie C, No. 137, Santiago de Chile, 1972.

Chackiel, J., Estructura de la fecundidad por edades. Ajuste y proyección mediante la función de Gompertz linealizada. En Notas de Población No. 20, agosto 1979.

Kandiah, V., The use of the relational fertility model parameters in population projections. East-West Center, Honolulu.

Martin, P., Une application des fonctions de Gompertz a l'étude de la fecondité d'une cohorte. En Population No. 6, 1967.

Spielman, E., Notas sobre la utilización de la función de Gompertz en el estudio de la fecundidad. En Notas de Población No. 12. CELADE, diciembre 1972. 